\title{
Construção e validação de um índice para o planejamento da mobilidade com foco em polos geradores de viagens
}

\author{
Angélica Meireles de Oliveira ${ }^{1}$ e Antônio Nélson Rodrigues da Silva²
}

\begin{abstract}
Resumo: Abordagens de planejamento destinadas a enfrentar os problemas de mobilidade associados aos grandes Polos Geradores de Viagens (PGVs) devem considerar as fases de diagnóstico, planejamento e avaliação das ações propostas. Esta é a estrutura da metodologia de sete etapas apresentada neste estudo, em que um índice de mobilidade sustentável é concebido para satisfazer necessidades locais. O objetivo deste artigo é discutir as diretrizes e procedimentos adotados para a construção e validação deste índice, estruturado em forma de um modelo hierárquico, bem como os resultados obtidos com a sua aplicação em um campus universitário. A abordagem permitiu a identificação e avaliação dos principais fatores que afetam as condições de mobilidade do campus, além da associação destes aos diferentes modos de transporte. A importância da estratégia de validação foi evidenciada pela identificação de lacunas na estrutura do índice. Alguns ajustes foram então sugeridos, o que parece ser essencial para fazer do índice uma ferramenta também mais efetiva de planejamento.

Palavras-chave: planejamento da mobilidade, polos geradores de viagem, mobilidade sustentável.
\end{abstract}

Abstract: Planning approaches designed to tackle mobility issues associated to large Trip Generators (TGs) must consider the stages of diagnosis, planning, and assessment of the proposed actions. This is the framework of the seven-step methodology introduced in this study, in which a sustainable mobility index is designed to meet the local needs. The objective of this paper is to discuss the guidelines and procedures adopted for the construction and validation of this hierarchical model, as well as the results obtained with an application in a university campus. The approach allowed the identification and assessment of the main factors affecting the mobility conditions of the campus, in addition to their association with the different modes of transport. The importance of the validation strategy was evidenced by the identification of gaps in the index structure. A few adjustments were then suggested, what seems essential for making the index a more effective planning tool.

Keywords: mobility planning, trip generators, sustainable mobility.

\section{INTRODUÇÃO}

Os problemas de mobilidade estão fortemente relacionados à forma de utilização do espaço público destinado ao deslocamento. O desafio é otimizar este espaço, quase sempre limitado, para atender à crescente demanda, influenciada pelas características do uso do solo. Assumindo-se que o sistema de transportes pode ser dividido em software (gestão) e hardware (infraestrutura), as estratégias de planejamento devem necessariamente abordar ambos os aspectos.

A complexidade dos problemas de mobilidade encontrados em centros urbanos é influenciada por diversos fatores, que variam de acordo com o cenário e com as necessidades individuais dos usuários. Em uma análise microscópica destes problemas, os Polos Geradores de Viagens (PGVs) representam pontos críticos. A fim de elaborar uma metodologia capaz de avaliar as condições de mobilidade e auxiliar no planejamento destes pontos, buscou-se uma abordagem multicritério que identifique os aspectos relevantes que contribuem, direta e indiretamente, para a mobilidade, além de avaliar o desempenho e interação da gestão e infraestrutura do sistema de transportes. Esta metodologia foi traduzida na forma de um índice, com foco na sustentabilidade, que expressa a qualidade da mobilidade em

\footnotetext{
1 Angélica Meireles de Oliveira, Departamento de Engenharia de Transporte, USP. (angelica_meireles@hotmail.com)

2 Antônio Nélson Rodrigues da Silva, Departamento de Engenharia de

Transporte, USP. (anelson@sc.usp.br)
}

Manuscrito recebido em 28/02/2016 e aprovado para publicação em $12 / 09 / 2016$

Este artigo é parte de TRANSPORTES v. 24, n. 3, 2016. ISSN: 2237-1346 (online). DOI:10.4237/transportes.v24i3.1081
PGVs, particularmente naqueles em que os deslocamentos internos são tão relevantes e complexos quanto os deslocamentos externos.

Porém, a construção de um índice que avalie os padrões de mobilidade é apenas uma etapa de uma metodologia mais abrangente, elaborada com a finalidade de nortear o diagnóstico e o planejamento, além de avaliar as medidas de intervenção aplicadas no sistema de mobilidade de PGVs. Deste modo, o objetivo deste trabalho é apresentar a metodologia proposta e discutir os procedimentos e diretrizes adotados para a construção e validação do índice. Além disso, visa discutir também os resultados obtidos em um estudo de caso realizado em um campus universitário. É importante destacar que o termo validação foi aqui empregado essencialmente para verificar a adequação do índice proposto ao contexto do caso estudado.

\section{REFERENCIAL TEÓRICO}

Os Polos Geradores de Viagens possuem a capacidade de modificar a organização dos centros urbanos e dos padrões de deslocamentos, pois geram centralidades, ou até mesmo subcentros, com a oferta de produtos/serviços (Kneib et al., 2012). O impacto dos PGVs exige uma reorganização do território urbano e, no longo prazo, a reestruturação da própria cidade em função dos padrões de acessibilidade que configurarão espacialmente a sua área de influência. Tais alterações e o dinamismo da estrutura urbana geram a necessidade de reavaliar e redefinir os papéis do transporte público, não motorizado e individual, a fim de favorecer a acessibilidade aos PGVs e a organização espacial urbana, por meio da adequação física do espaço viário e de estratégias de gerenciamento de mobilidade. Estas estratégias são constituídas por um conjunto de ferramentas 
de suporte e estímulos para a mudança de atitude e comportamento em relação ao uso de modos de transportes sustentáveis e a redução da dependência de fontes de energia não renováveis (Ferreira e Silva, 2008). Por se tratar de um processo de conscientização e educação, são aplicáveis a PGVs de diferentes tamanhos e/ou naturezas. Estas estratégias de gerenciamento podem ser baseadas nas boas práticas aplicadas em outros países, como observado por Wilhelm e Posch (2003) e Ferreira e Silva (2008).

A identificação das características a serem trabalhadas no PGV deve envolver uma etapa de diagnóstico. Uma ferramenta bastante utilizada para avaliar a qualidade da mobilidade e que também auxilia a definição de diretrizes de planejamento de sistemas complexos é a abordagem multicritério. Costa (2008), por exemplo, elaborou, a partir desta abordagem, uma metodologia de avaliação e planejamento da mobilidade sustentável em centros urbanos. No caso de PGVs, Oliveira et al. (2014) elaboraram um conjunto de indicadores para diagnosticar e sugerir medidas de intervenção para um campus universitário.

De acordo com Parra (2006), um campus universitário é, em geral, um PGV, que influencia e é influenciado pelas condições da cidade em que se insere. As universidades, como outros tipos de instituições públicas e privadas, quando localizados em uma cidade, têm impactos positivos e negativos sobre a área onde eles estão situados. Por um lado, contribuem para o prestígio da área; por outro lado, são grandes geradores de tráfego. A capacidade de equilibrar com sucesso os prós e contras da localização urbana destas grandes instituições geradoras de tráfego é crucial para o seu sucesso e para a habitabilidade da cidade (Danielis e Rotaris, 2014). Além disso, as Instituições de Ensino Superior (IES) têm um papel preponderante na discussão da mobilidade sustentável no âmbito social, econômico e ambiental, uma vez que representam centros de inovação. Segundo Parra e Portugal (2007), o ambiente do campus, ao promover a formação e educação de pessoas, reúne condições favoráveis ao gerenciamento da mobilidade e à extensão desta proposta ao resto da sociedade. Com este perfil, forma e educa futuros profissionais e tomadores de decisão, que desempenharão um papel ativo e de responsabilidade perante a sociedade. Nesta fase de formação é importante a difusão das práticas de gestão de mobilidade, com o intuito de conscientizar a comunidade acadêmica (Balsas, 2003; Ferreira et al., 2013)

Como observado por Ferreira e Silva (2008), na União Europeia, a elaboração e aplicação de medidas de intervenções foram norteadas a partir da caracterização do perfil de viagens às IES. Porém, a avaliação posterior foi realizada através da redução das taxas de poluição e consumo de energia. Em nenhum dos casos estudados por Ferreira e Silva (2008), no entanto, a metodologia usada foi a de planejamento baseado em indicadores, como proposto neste trabalho.

No Brasil, pesquisas relacionadas a campi universitários têm, em geral, abordado as condições de mobilidade de forma isolada, avaliando condições e situações mais pontuais. Dentre os trabalhos consultados, alguns estudos avaliaram as condições das calçadas e/ou acessibilidade nos campi e apontaram deficiências em instalações consolidadas ou recentemente construídas (Cotrim et al., 2012; Chaves e Duarte, 2013; Aguiar et al., 2009; Tobias et al., 2013;
Correia, 2015). Em outras abordagens, pesquisas investigaram a necessidade da priorização de modos não motorizados e a utilização de medidas de gerenciamento de mobilidade, como forma de estimular a utilização de modos de transporte mais sustentáveis (Neri e Costa, 2014; Parra e Portugal, 2007).

Já em estudos um pouco mais abrangentes foram investigadas as barreiras e motivações relacionadas com a escolha do modo de transporte, bem como a possibilidade de os usuários adotarem modos de transporte mais sustentáveis (Sanches e Ferreira, 2013; Stein, 2013; Hu e Schneider, 2015). Como resultado, estes trabalhos apontaram, nos campi, problemas de diferentes naturezas e intensidades, envolvendo infraestrutura, gestão, níveis de conscientização e, principalmente, situações de insegurança apontadas como fatores que incentivam indiretamente o uso de veículos motorizados.

Apesar de estudos abordarem os problemas observados nos campi universitários, ainda há uma carência no que tange ao planejamento, pois muitas universidades ainda não possuem planos efetivos para gestão da mobilidade. Em alguns casos, planos desta natureza encontram-se em processo de elaboração. Porém, para que este planejamento seja feito de forma eficaz e eficiente são necessárias ferramentas que possibilitem uma visão holística dos problemas de mobilidade que envolvem o campus. Alguns dos fatores que influenciam o desenvolvimento da mobilidade são internos, e dependem diretamente da gestão e infraestrutura do campus, outros são externos e estão relacionados com questões econômicas, políticas e sociais. Tão importante quando identificar estes fatores é quantificar a influência de cada um deles no desenvolvimento da mobilidade. Visando esta abordagem holística dos campi universitários é que foi proposta a metodologia multicritério aqui apresentada, conforme detalhado na próxima seção deste trabalho.

\section{METODOLOGIA}

A metodologia proposta, que visa promover o planejamento da mobilidade de Polos Geradores de Viagens com foco na sustentabilidade, organiza-se em sete etapas e de forma cíclica, como mostrado na Figura 1.

No diagnóstico da mobilidade (etapa $i$ ), deve ser realizado um levantamento que permita caracterizar os padrões de deslocamento e investigar o potencial de mudança de comportamento dos diferentes grupos de usuários (definido de acordo com o público alvo do PGV). Este levantamento pode ser realizado por meio de questionários on-line, como na pesquisa realizada por Stein (2013) em um campus universitário. Os resultados desta etapa devem propiciar a identificação dos fatores determinantes para a escolha do modo de deslocamento. Na etapa $i i$, estes fatores devem ser classificados como medidas (possíveis soluções sugeridas no questionário) ou indicadores, e posteriormente agrupados em níveis hierárquicos (DOMÍNIOS, Temas e Indicadores, por exemplo), de acordo com suas características (Costa, 2008). Fatores que envolvem diretamente medidas de intervenção também podem ser associados a um indicador, embora só sejam analisados na etapa vi, que envolve a elaboração destas medidas. A atribuição dos pesos pode ser feita, 

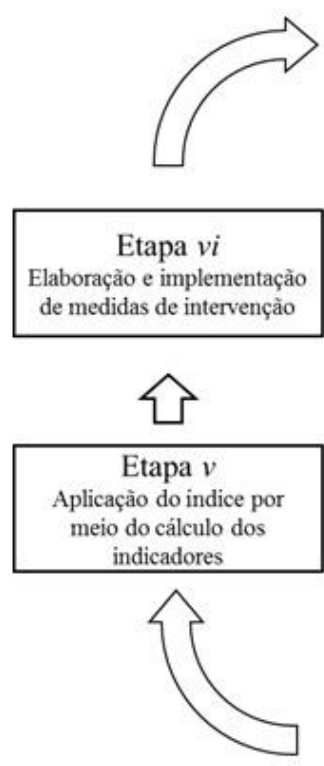
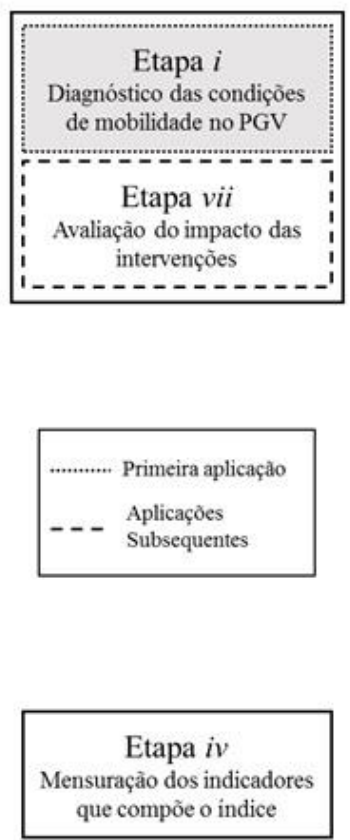

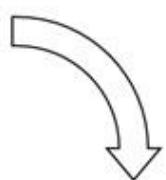

Etapa $i i$

Elaboraçào ou adaptação do

indice de mobilidade

sustentável para o PGV
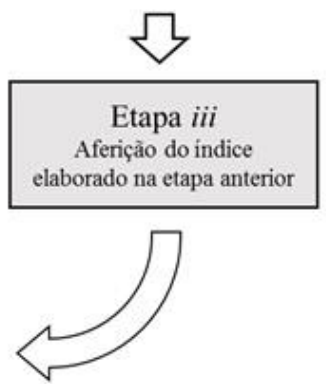

Etapas da metodologia discutidas neste artigo

Figura 1. Fluxograma das etapas da metodologia proposta

por exemplo, de acordo com a frequência com que o fator é citado pelos respondentes (como proposto em Oliveira e Rodrigues da Silva, 2014), por meio de um painel de especialistas (Costa, 2008) ou pela atribuição de pesos iguais aos indicadores (Olszak, 2012).

Apesar da etapa vi não ser abordada no presente artigo, é importante destacar que, após a aplicação do índice, a metodologia propõe a avaliação das sugestões associadas a cada indicador como potenciais soluções para os problemas de mobilidade do PGV. Por exemplo, se alguns dos indicadores calculados ficarem aquém da condição esperada ou desejada, as sugestões obtidas na etapa $i i$ que estiverem relacionadas com estes indicadores serão consultadas e analisadas, com o objetivo de melhorar as condições de mobilidade e incorporar ao processo de planejamento a visão dos usuários consultados.

Para a validação do índice obtido (etapa iii) deverão ser realizadas atividades (como workshops com grupos focais, por exemplo) envolvendo os diferentes tipos de usuários ou grupo(s) de representantes, a fim de avaliar a relevância e aplicabilidade dos indicadores em relação à mobilidade sustentável do PGV. Na etapa $i v$, devem ser definidos os métodos de mensuração e os valores de referência para normalização de cada indicador (por exemplo, em uma escala de 0 a 1, em que zero representa uma péssima avaliação e um, a melhor avaliação possível). A etapa seguinte, a de aplicação do índice, envolve a coleta de dados para o cálculo dos indicadores. Este resultado e as sugestões obtidas na pesquisa de opinião, associadas aos indicadores na etapa $i i$, servirão como base para a elaboração da proposta de intervenção (etapa vi), destacando as soluções de maior viabilidade e impacto para a mobilidade. Na etapa vii, sugerese a reaplicação do índice para avaliar as mudanças observadas após um intervalo de dois a cinco anos. A ferramenta proposta também pode ser utilizada para comparar a qualidade da mobilidade sustentável entre PGVs semelhantes.

Na primeira aplicação devem ser executadas todas as etapas que compõem a metodologia. Porém, nas aplicações subsequentes, algumas etapas não precisam ser realizadas. Outras podem ser realizadas de forma simplificada. No caso de uma aplicação subsequente, a avaliação dos impactos das intervenções realizadas (etapa vii) será, por consequência, também o diagnóstico das condições de mobilidade do PGV (etapa $i$ ). Da mesma maneira, a etapa $i i$ consistirá apenas na adaptação do índice elaborado na primeira aplicação, visando à obtenção de um índice que represente o novo cenário do PGV. Já as etapas $i i i, v, v i$ e vii devem ser executadas de forma similar à primeira aplicação. No tocante à etapa $i v$, está só deve ser realizada se houver a inserção de novos indicadores no índice proposto, ou a necessidade de alterar os valores de referência.

\section{ESTUDO DE CASO}

A aplicabilidade da metodologia de planejamento da mobilidade de PGV proposta está sendo testada em um campus universitário. O campus selecionado é formado por duas áreas, com uma população de aproximadamente 9.000 usuários regulares (USP, 2015), que representam cerca de $4 \%$ da população da cidade em que está situado (IBGE, 2010). A escolha do cenário de estudo foi motivada, entre outras coisas, pela necessidade de concepção de um plano de mobilidade do campus.

A primeira etapa, de diagnóstico da mobilidade do campus, baseou-se na coleta de dados realizada no ano de 2012, por Stein (2013). Esta pesquisa foi aplicada por meio de questionários on-line, enviados por $e$-mail a todos os usuários do campus, resultando em 2.226 questionários válidos. Para o diagnóstico do campus, foram utilizadas as duas questões abertas contidas na pesquisa, através das quais se coletou a opinião dos usuários em relação aos aspectos de mobilidade do campus. Nesta etapa, foram obtidas 1.807 respostas válidas, entre ambas as questões (em média 900 respondentes por questão), representando contribuições de aproximadamente $10 \%$ da população do campus. 


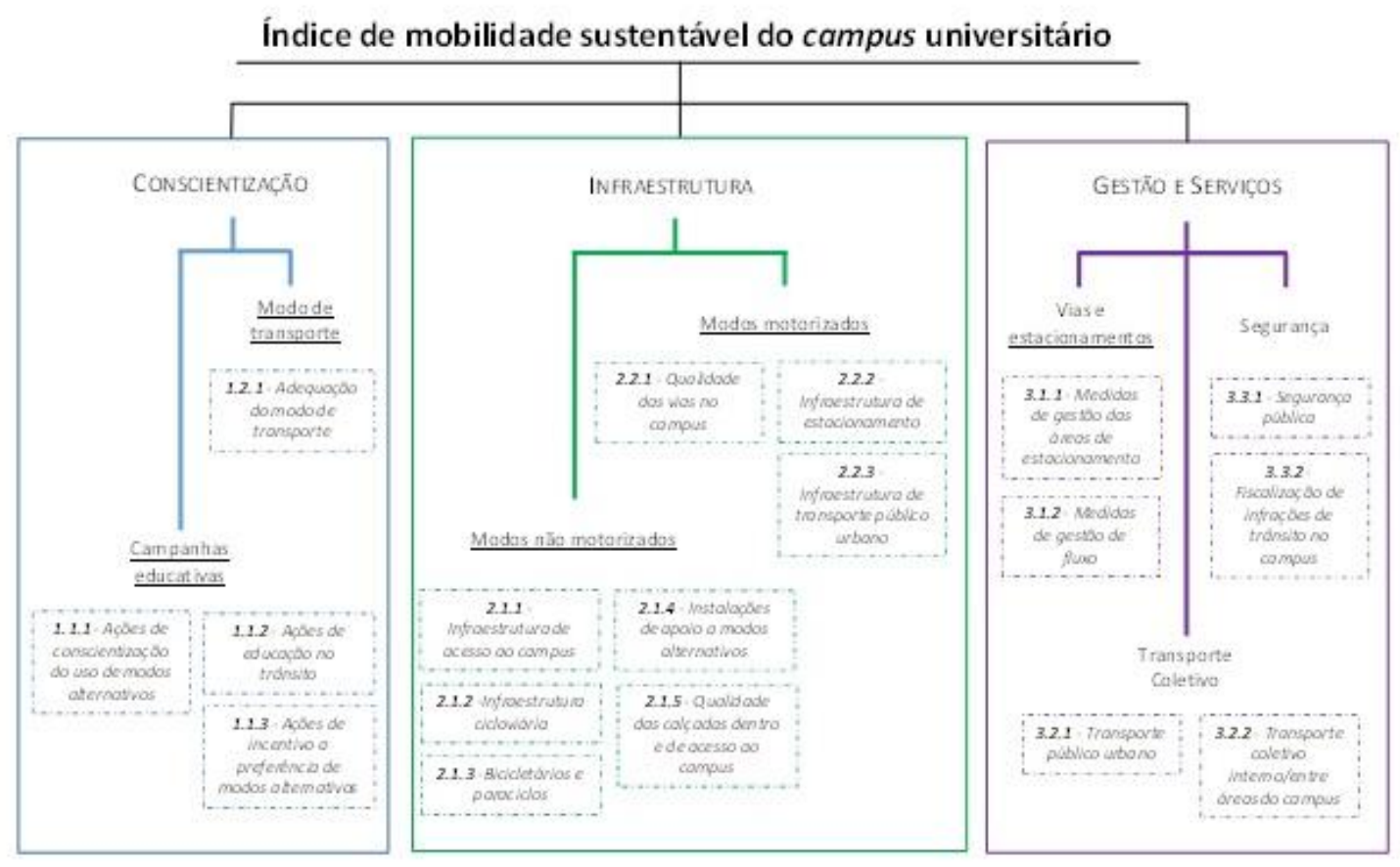

Figura 2. Índice obtido na etapa ii da metodologia proposta

Na etapa $i i$, analisou-se o conjunto de respostas, conforme descrito na metodologia. Após agrupar em níveis hierárquicos e classificar os aspectos em indicadores ou medidas (por exemplo, "rodízio de placas para o uso do estacionamento" é uma medida de gestão, que está contemplada no indicador "Medidas de gestão das áreas de estacionamento"), foram obtidos 48 indicadores. Optou-se por excluir, do índice, os indicadores que apresentaram menos de $1 \%$ de frequência nas respostas. Desta forma, foi obtido um índice com 18 Indicadores (que representaram 96\% da frequência total de respostas do questionário), distribuídos em 7 (sete) Temas e 3 (três) Domínios. A estrutura hierárquica e o conjunto de indicadores obtidos nesta etapa estão representados na Figura 2.

Após a definição do Índice para planejamento da Mobilidade Sustentável em um Campus Universitário (IMSCamp) pôde ser realizada a etapa iii, que consiste na validação deste índice. A metodologia proposta sugere a realização de consulta aos usuários e/ou a seus representantes. A fim de testar a abordagem sugerida neste trabalho, optou-se por realizar os dois tipos de validação. A consulta aos usuários foi realizada através da aplicação de um novo questionário. Por meio de questão aberta, os respondentes explanaram sobre as necessidades que deveriam ser abordadas no plano de mobilidade que está em vias de ser desenvolvido para o campus. Os resultados desta nova pesquisa foram comparados aos fatores contemplados na versão original do IMSCamp (conforme a Figura 2).

Uma segunda etapa do processo de validação ocorreu com o auxílio da comissão constituída para elaborar o Plano de Mobilidade do campus, composta por representantes de alunos, servidores docentes e técnico-administrativos. Neste caso, foram realizadas reuniões quinzenais para a validação, nas quais se discutiu desde o processo de elaboração dos indicadores até a concepção do índice final (apresentado na Tabela 1). Com o diagnóstico elaborado a partir de elementos apontados pelos usuários e a realização destas duas atividades de validação, a metodologia elaborada destaca a interface entre planejadores e usuários, por meio da promoção de um processo de planejamento participativo. De forma a atingir o objetivo proposto para este trabalho, uma vez apresentada a metodologia, pode-se discutir os procedimentos e diretrizes para a construção do IMSCamp, os processos de validação, bem como os resultados obtidos no estudo de caso. Os resultados dessas análises são mostrados na seção seguinte.

\section{RESULTADOS}

Com o objetivo de investigar a adequação do Índice de Mobilidade Sustentável em um Campus Universitário (IMSCamp) para o cenário em que foi concebido, foram realizadas duas análises principais. A primeira tem foco na consistência da validação do índice realizada pelos usuários, enquanto que a segunda está relacionada à capacidade do índice de abordar os diferentes modos de transportes que compõem o quadro de mobilidade do campus.

A concepção dos indicadores baseou-se na opinião e percepção dos usuários. Deste modo, é razoável afirmar que o índice reflete as condições do cenário. Porém, o levantamento utilizado no processo de construção da estrutura original do índice foi realizado em 2012. Com o objetivo de comparar as necessidades atuais e validar o índice, uma nova consulta foi realizada em 2014. O levantamento de 2014 também foi conduzido por meio de questão aberta aplicada aos usuários. Porém, neste caso, adotou-se o princípio do método de análise documental, o qual propõe investigar o material textual a partir da busca de palavraschave (Bardin, 2011). Esta busca foi auxiliada pela ferramenta chamada "word clouds" ou "nuvens de palavras", que se baseia na contagem de palavras do texto, retornando com as de maior frequência. Este mesmo procedimento foi aplicado ao levantamento de 2012. Dentre os resultados foram filtradas as palavras-chave de maior frequência, a fim de realizar uma comparação entre os levantamentos e os indicadores propostos, como mostrado na Figura 3. 


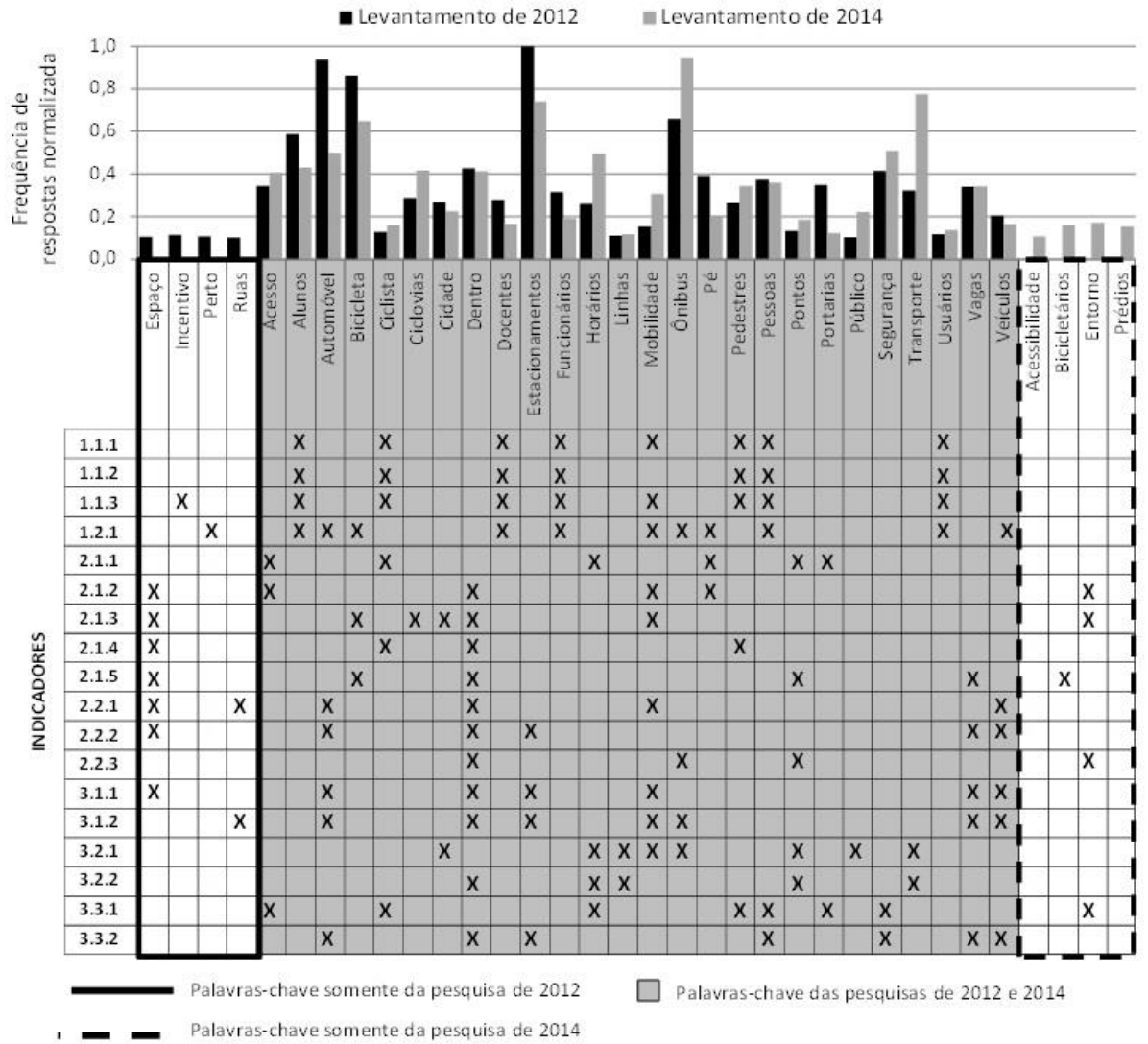

Figura 3. Palavras-chave encontradas, com maior frequência, nos levantamentos de 2012 e 2014, comparadas aos indicadores propostos na Figura 2

Na comparação entre os levantamentos, foram analisadas as 30 palavras-chave de maior frequência. Destas, 26 foram comuns a ambas as pesquisas, ou seja, $87 \%$ das palavras-chave se repetiram. Posteriormente, cada palavrachave foi associada aos indicadores propostos na etapa anterior. A associação foi realizada de acordo com a definição e abrangência de cada indicador. Na Figura 3, as palavraschave comuns entre os levantamentos estão sombreadas, enquanto que as contornadas por linhas continuas e tracejadas são as palavras encontradas com maior frequência nos levantamentos de 2012 e 2014, respectivamente.

Como mostrado na Figura 3, das 30 palavras-chave encontradas em ambas às pesquisas, apenas 2 não estão contempladas pelos indicadores, sendo estas "Acessibilidade" e "Prédios". Analisando o conjunto de respostas, pode se constatar que ambas as palavras referiam-se às condições de acessibilidade universal. Estas palavras pertencem ao levantamento de 2014 e não tiveram frequência expressiva no levantamento de 2012, uma das razões pelas quais não estavam contempladas no índice. Outro viés na elaboração do IMSCamp, responsável por esta lacuna, é a abordagem utilizada no questionário de 2012, que tinha como objetivo apenas obter um diagnóstico da mobilidade para o campus. As perguntas abertas estavam focadas nas necessidades dos usuários em relação à mobilidade sustentável. Uma vez que poucos usuários possuem algum tipo de restrição física extrema de mobilidade, este assunto foi pouco citado entre as respostas. Já na pesquisa de 2014, o foco é a elaboração do índice que auxiliará o planejamento de mobilidade no campus, bem como a elaboração do plano de mobilidade. Neste levantamento, a questão aberta utilizada para validação foi portanto direcionada aos aspectos, que segundo os usuários, deveriam ser contemplados nas diretrizes de planejamento da mobilidade do campus.

A partir do resultado obtido no processo de validação, que evidenciou uma lacuna na abrangência do conjunto de indicadores, a estrutura mostrada na Figura 1 foi reformulada. Para sanar a deficiência identificada no índice incluiu-se no Domínio "Infraestrutura", o Tema "Acessibilidade". Neste novo Tema, o indicador "Qualidade das calçadas dentro e de acesso ao campus" foi adaptado de forma a avaliar também as condições de acessibilidade das calçadas. Além disso, dois novos indicadores foram incluídos, visando avaliar as demais necessidades dos usuários com restrição de mobilidade: "Vagas para usuários com restrição de mobilidade" $\mathrm{e}$ "Acessibilidade de prédios". A estrutura final do IMSCamp, bem como uma breve descrição dos indicadores, é apresentada na Tabela 1.

A segunda análise realizada teve como objetivo verificar a abrangência do IMSCamp, relacionando ao conjunto de indicadores os modos de transporte (a pé, bicicleta, transporte coletivo, transporte de carga e veículos motorizados de uso privado) utilizados com maior frequência pelos usuários do campus, como mostra a Figura 4. Observa-se que, exceto o transporte de carga, cada grupo está associado a 9 (nove) ou 10 (dez) indicadores, sendo estes distribuídos entre os três domínios (Conscientização, Infraestrutura e Gestão e Serviços). Esta característica evidencia que o IMSCamp abrange de forma balanceada as necessidades dos quatro grupos (a pé, bicicleta, transporte coletivo e transporte motorizado de uso privado). 
OLIVEIRA, A.M.; RODRIGUES DA SILVA, A.N.

Tabela 1. Indicadores que compõem o IMSCamp - Índice para o planejamento da Mobilidade Sustentável em um Campus Universitário

\begin{tabular}{|c|c|c|c|}
\hline Domínio & Tema & Indicador & \begin{tabular}{|l} 
Definiçãa \\
\end{tabular} \\
\hline \multirow{4}{*}{ 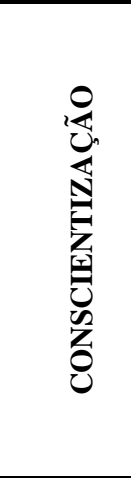 } & \multirow{4}{*}{ 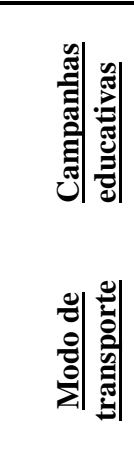 } & $\begin{array}{c}\text { Ação de conscientização do uso de modos } \\
\text { alternativos }\end{array}$ & $\begin{array}{c}\text { Abrangência e eficácia das ações de } \\
\text { conscientização do uso de modos alternativos } \\
\text { (benefícios para a saúde, redução da emissão de } \\
\text { poluentes, etc.) }\end{array}$ \\
\hline & & Ação de educação no trânsito & $\begin{array}{c}\text { Abrangência e eficácia das ações de educação } \\
\text { de trânsito para usuários do campus }\end{array}$ \\
\hline & & $\begin{array}{c}\text { Ação de incentivo à utilização de modos mais } \\
\text { sustentáveis }\end{array}$ & $\begin{array}{l}\text { Abrangência e qualidade de ações de incentivo } \\
\text { à preferência de modos mais sustentáveis } \\
\text { (descontos, bonificação para usuários que não } \\
\text { utilizam carro, etc.) }\end{array}$ \\
\hline & & Adequação do modo de transporte & $\begin{array}{l}\text { Grau de adequação da escolha do modo de } \\
\text { transporte em função da distância a ser } \\
\text { percorrida }\end{array}$ \\
\hline \multirow{10}{*}{ 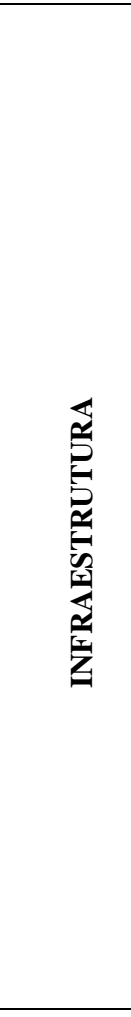 } & \multirow{4}{*}{ 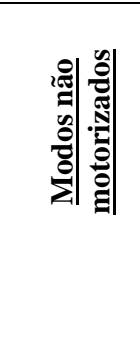 } & Infraestrutura de acesso ao campus & $\begin{array}{l}\text { Qualidade, localização e número de entradas do } \\
\text { campus disponíveis para pedestres e ciclistas }\end{array}$ \\
\hline & & Infraestrutura cicloviária & $\begin{array}{c}\text { Extensão, qualidade e localização da } \\
\text { infraestrutura, próximo e dentro do campus }\end{array}$ \\
\hline & & Bicicletários e paraciclos & $\begin{array}{c}\text { Número, distribuição, localização, estado de } \\
\text { conservação e segurança dos bicicletários e } \\
\text { paraciclos dentro do campus }\end{array}$ \\
\hline & & Instalações de apoio a modos alternativos & $\begin{array}{l}\text { Disponibilidade, localização e qualidade de } \\
\text { vestiários, bebedouros, guarda-volumes, etc. }\end{array}$ \\
\hline & \multirow{3}{*}{ 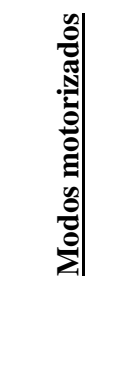 } & Qualidade das vias no campus & $\begin{array}{l}\text { Avaliação das condições do pavimento e da } \\
\text { sinalização vertical e horizontal }\end{array}$ \\
\hline & & Infraestrutura de estacionamento & $\begin{array}{c}\text { Capacidade das áreas de estacionamento para } \\
\text { atender à demanda }\end{array}$ \\
\hline & & Infraestrutura de transporte público urbano & $\begin{array}{c}\text { Avaliação do acesso aos pontos de ônibus } \\
\text { (distância, iluminação, segurança, etc.) e aos } \\
\text { pontos de recarga do passe escolar }\end{array}$ \\
\hline & \multirow{3}{*}{ 寄 } & $\begin{array}{c}\text { Qualidade das calçadas dentro e de acesso ao } \\
\text { campus }\end{array}$ & $\begin{array}{l}\text { Largura, adequadas da manutenção dos } \\
\text { calçamentos e condições de acessibilidade nas } \\
\text { calçadas internas e no perímetro do campus }\end{array}$ \\
\hline & & $\begin{array}{c}\text { Vagas para usuários com restrição de } \\
\text { mobilidade }\end{array}$ & $\begin{array}{l}\text { Disponibilidade e localização das vagas de } \\
\text { estacionamento para usuários com restrição de } \\
\text { mobilidade permanente e idosos }\end{array}$ \\
\hline & & Acessibilidade dos prédios & $\begin{array}{c}\text { Avaliação qualitativa da acessibilidade } \\
\text { dos prédios }\end{array}$ \\
\hline \multirow{6}{*}{ 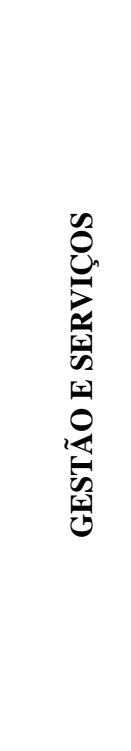 } & \multirow{2}{*}{ 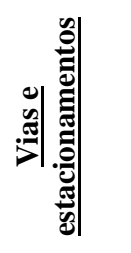 } & $\begin{array}{c}\text { Medidas de gestão das áreas de } \\
\text { estacionamento }\end{array}$ & $\begin{array}{l}\text { Eficiência das medidas de gestão do } \\
\text { estacionamento (rodizio, vagas reservadas, etc.) }\end{array}$ \\
\hline & & Medidas de gestão de fluxo & Eficiência das medidas de gestão do fluxo \\
\hline & \multirow{3}{*}{ 氡: } & Transporte público urbano & $\begin{array}{l}\text { Qualidade dos serviços de transporte público } \\
\text { urbano (frequência, lotação, pontualidade, etc.) }\end{array}$ \\
\hline & & $\begin{array}{c}\text { Transporte coletivo interno/entre áreas do } \\
\text { campus }\end{array}$ & $\begin{array}{c}\text { Qualidade dos serviços de transporte oferecido } \\
\text { pela universidade (frequência, lotação, } \\
\text { pontualidade, etc.) }\end{array}$ \\
\hline & & Segurança pública & $\begin{array}{l}\text { Sensação de segurança no acesso e dentro do } \\
\text { campus, em diferentes turnos }\end{array}$ \\
\hline & 氚 & $\begin{array}{c}\text { Fiscalização de infrações de trânsito no } \\
\text { campus }\end{array}$ & $\begin{array}{l}\text { Sensação de segurança no acesso e dentro do } \\
\text { campus, em diferentes turnos } \\
\text { Eficácia da fiscalização do uso de } \\
\text { estacionamento dentro do campus (capacitação } \\
\text { dos funcionários, sistema de notificação, } \\
\text { medidas de advertência, etc.) }\end{array}$ \\
\hline
\end{tabular}




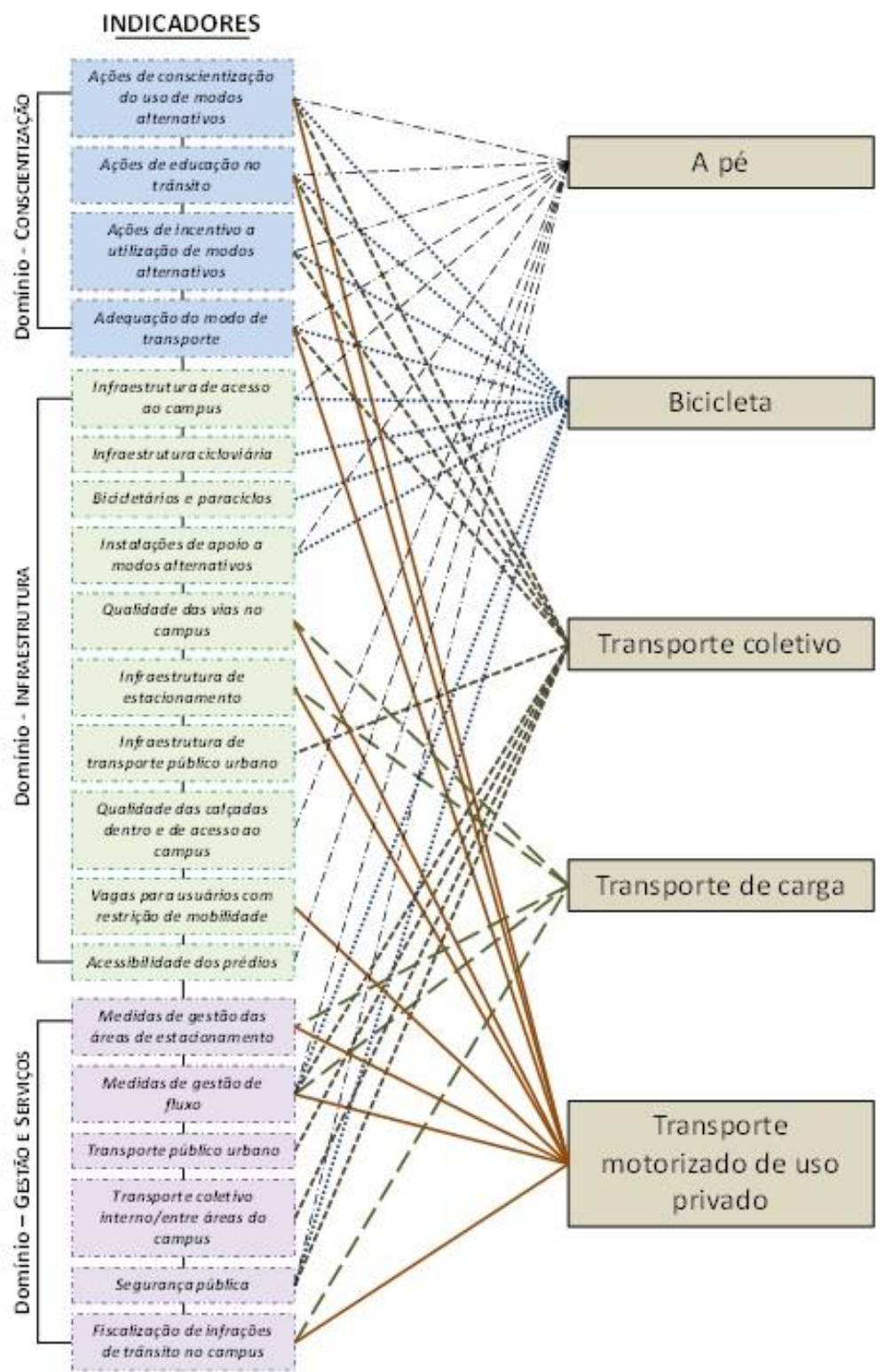

Figura 4. Associação do conjunto de indicadores aos cinco principais modos de transportes utilizados no campus universitário

\section{CONCLUSÃO}

Com o objetivo de contribuir para o desenvolvimento de uma metodologia para diagnóstico e planejamento da mobilidade em PGV's, este trabalho discute os resultados obtidos a partir de um estudo de caso desenvolvido em um campus universitário. A metodologia proposta é composta de sete etapas. Neste trabalho, porém, discutem-se apenas duas etapas (elaboração e validação do índice para o planejamento da mobilidade sustentável), através de sua aplicação em um estudo de caso.

A partir da aplicação realizada no estudo de caso, duas conclusões principais podem ser destacadas. A primeira se refere especificamente ao caso estudado, no qual foram identificados problemas no índice originalmente proposto, verificados durante o processo de validação, enquanto que a segunda diz respeito ao resultado da metodologia.

Quanto à validação no caso estudado, embora os indicadores elaborados descrevessem as necessidades dos usuários, o que foi comprovado a partir da comparação entre a definição e abrangência dos indicadores e as palavras- chave mais frequentes no questionário aplicado no ano de 2012, o mesmo não se pode dizer para o questionário mais recente, que reflete melhor o momento atual. Duas palavraschave referentes à acessibilidade das dependências do campus não estavam relacionadas aos indicadores. Estas lacunas no IMSCamp só foram evidenciadas a partir de um levantamento com perguntas específicas sobre as necessidades para a criação do plano de mobilidade para o campus em 2014. Desse modo, conclui-se que é fundamental realizar um levantamento específico na etapa de diagnóstico, visando a elaboração e aplicação de um índice dessa natureza.

No tocante à metodologia, pôde-se concluir, a partir do estudo de caso, que a abordagem proposta permitiu identificar e avaliar os principais fatores que afetam os padrões de mobilidade do PGV, de forma abrangente e balanceada entre os diferentes modos de transportes. A aplicação possibilitou concluir ainda que a etapa de validação é de extrema importância, pois permite reavaliar o índice e corrigilo antes de seu uso como ferramenta efetiva de planejamento. Neste aspecto, cabe discutir o fato de que questionários gerais de caracterização dos padrões vigentes de mobilidade são úteis, mas como elemento de partida para a construção do índice. Eles podem, no entanto, ocultar elementos 
fundamentais para o planejamento efetivo da melhoria das condições de mobilidade, que eventualmente só são identificadas em consultas direcionadas para este fim específico.

\section{AGRADECIMENTOS}

Os autores agradecem ao CNPq (Conselho Nacional de Desenvolvimento Científico e Tecnológico) e à Universidade de São Paulo pelo apoio para a realização da pesquisa.

\section{REFERÊNCIAS}

Aguiar, F. O.; R. A. R. Ramos e A. N. Rodrigues da Silva (2009) Cenários de Avaliação das Restrições à Mobilidade dos Pedestres em Espaços Públicos: o Caso de um Campus Universitário. Revista Transportes, v. 17, n. 2, p. 5-15.

DOI: $10.14295 /$ transportes.v17i2.354

Balsas, C. J. (2003) Sustainable Transportation Planning on College Campuses. Transport Policy, v. 10, n. 1, 35-49. DOI: $10.1016 / \mathrm{S} 0967-070 \mathrm{X}(02) 00028-8$

Bardin, L. (2011) Análise de Conteúdo. Traduzido por: Reto, L. A., Pinheiro, A. Edições 70. Lisboa.

Chaves, T. S. e P. C. Duarte (2013) Acessibilidade e Mobilidade: o Campus da Universidade Federal do Pampa - Bagé/RS. Anais do XXVII Congresso de Pesquisa e Ensino em Transportes, ANPET, Belém. Disponível em: < http://www.anpet.org.br/ssat/interface/content/autor/trabalhos/pu blicacao/2013/384_RIC.pdf >. Acesso em 15 jul 2014.

Correia, S. A. V. L. (2015) Atributos de Rede para Pedestres com Restrições de Mobilidade em um Modelo para Avaliação da Acessibilidade. Dissertação de Mestrado, Universidade de São Paulo, São Carlos. DOI 10.11606/D.18.2015.tde-13062015143424

Costa, M. D. S (2008). Um índice de Mobilidade Urbana Sustentável. Tese de Doutorado, Universidade de São Paulo. São Carlos. DOI 10.11606/T.18.2008.tde-01112008-200521

Cotrim, S. L.; M. E. P. Assunção, F. A. Simões e D. A. Medeiros Filho (2012) Qualidade das Calçadas no Campus da Universidade Estadual de Maringá - PR. Anais do III Simpósio de Pós-graduação em Engenharia Urbana, Maringá. Disponível em: <

http://www.eventos.uem.br/index.php/simpgeu/simpgeu/paper/vi ew/922 >. Acesso em 11 jul 2014.

Danielis, R. e L. Rotaris, (2014) The impact of transportation demand management policies on commuting to college facilities: A case study at the University of Trieste, Italy. Transportation Research Part A: Policy and Practice, v. 67, 127-140. DOI: $10.1016 /$ j.tra.2014.06.011

Ferreira, D. e J. P. Silva (2008). Mobilidade Sustentável em Campi Universitários - Boas Práticas Europeias. Relatório de projeto, Escola Superior de Gestão e Tecnologia, Instituto Politécnico de Leiria. Disponível em: <

https://issuu.com/rgiaretta/docs/mob_sust_campus_univ_boas_pr at_eu >. Acesso em 30 mai 2014.

Ferreira, D.;J. P. Silva e A. B. Silva (2013) Impactos dos Modos de Transporte Sustentáveis em Instituições de Ensino Superior o Caso do Instituto Politécnico de Leiria. Anais do $7^{\circ}$ Congresso
Rodoviário Português - "Novos desafios para a atividade rodoviária", CRP, Lisboa. Disponível em: < http://crp.pt/docs/A45S140-77_Art_T2_7CRP_2013.pdf > Acesso em: 15 jul 2014.

Hu, L. e R. J. Schneider (2015) Improving university transportation sustainability: reducing barriers to campus bus and bicycle commuting. International Journal of Sustainability Policy and Practice, v. 11, n. 1, 24-34. Disponível em: <

http://ijspp.cgpublisher.com/product/pub.274/prod.91 >. Acesso em: 6 jun de 2016 .

IBGE (2010). Censo Demográfico 2010. Instituto Brasileiro de Geografia e Estatística. Disponível em:

<http://www.ibge.gov.br/>. Acesso em: 14 jul. 2014.

Kneib, E. C.; G. M. I. Tedesco; A. P. B. G. Barros e M. Paiva (2012) PGVs e Centralidades: Impactos na Escala Urbana e Metropolitana. In: Licínio da Silva Portugal. (Org.). Polos Geradores de Viagens Orientados à Qualidade de Vida e Ambiental. $1^{a}$ ed. Rio de Janeiro: Interciência, p. 671-704.

Neri, H. C. F. R. e A. D. L. Costa (2014) Transporte Não Motorizado: o Uso e Potencialidade da Bicicleta no Entorno do Campus da Universidade Federal do Amazonas - UFAM. Anais do XXVIII Congresso de Pesquisa e Ensino em Transportes, ANPET, Curitiba. Disponível em: < http://www.anpet.org.br/xxviiianpet/anais/documents/RT429.pdf >. Acesso em: 15 jul de 2015

Oliveira, A. M. e A. N. Rodrigues da Silva (2014) Planejamento da Mobilidade com Foco em Grandes Polos Geradores de Viagens. Anais do XXVIII Congresso de Pesquisa e Ensino em Transportes, ANPET, Curitiba. Disponível em: < http://www.anpet.org.br/xxviiianpet/anais/documents/RT101.pdf >. Acesso em 15 jul de 2015.

Oliveira, A. M.; T. C. Ramos e A. N. Rodrigues da Silva (2014) Introduzindo Conceitos de Mobilidade Sustentável no Ensino Superior a Partir de Problemas de Mobilidade do Campus. Anais do PLURIS - $6^{\circ}$ Congresso Luso-Brasileiro para o Planejamento Urbano, Regional, Integrado e Sustentável. Lisboa, Portugal.

Olszak, E. (2012) Composite Indicators for a Sustainable Campus - Design Rationale and Methodology: The case of the Catholic Institute of Lille. Ecological Indicators, Lille, França, v. 23, p. 573-577. DOI: 10.1016/j.ecolind.2012.05.021

Parra, M. C. (2006) Gerenciamento da Mobilidade em Campus Universitários: Problemas, Dificuldades e Possíveis Soluções no Caso da Ilha do Fundão - UFRJ. Dissertação de Mestrado, Universidade Federal do Rio de Janeiro, Rio de Janeiro. Disponível em: < http://objdig.ufrj.br/60/teses/coppe_m/MarselaCaipaParra.pdf $>$. Acesso em: 15 jul de 2014.

Parra, M. C. e L. S. Portugal (2007) Estratégia de Gerenciamento da Mobilidade para um Campus Universitário: Caso UFRJ. Programa de Engenharia de Transportes (PET - COPPE/UFRJ), Universidade Federal do Rio de Janeiro, Rio de Janeiro. Disponível em <

http://redpgv.coppe.ufrj.br/arquivos/Marsela_Parra_Portugal_An pet_2007_Ger_Mob.pdf >. Acesso em 15 jul de 2014.

Sanches, S. P. e M. A. G. Ferreira (2013) Mobilidade em um Campus Universitário. Anais do $19^{\circ}$ Congresso Brasileiro de Transportes e Trânsito. Brasília. 
Stein, P. P. (2013) Barreiras, Motivações e Estratégias para Mobilidade Sustentável no Campus São Carlos da USP.

Dissertação de Mestrado, Universidade de São Paulo, São

Carlos. DOI: 10.11606/D.18.2013.tde-27062013-163702

Tobias, M. S. G.; A. M. Borges e A. N. R. Brito (2013) Desafios e Soluções para Mobilidade em Campus Universitário: um Estudo de Caso na UFPA - Belém - PA. Anais do XXVII

Congresso de Pesquisa e Ensino em Transportes, ANPET, Belém. Disponível em: <

http://www.anpet.org.br/ssat/interface/content/autor/trabalhos/pu blicacao/2013/363_CT.pdf >. Acesso em: 15 jul de 2014

USP São Carlos (2015) Sobre o Campus da USP em São Carlos. Disponível em:

<http://www.saocarlos.usp.br/index.php?option=com_content\&ta sk=view\&id=61\&Itemid=87> . Acesso em: 12 maio 2015 .

Wilhelm A. e K. -H. Posch (2003) Mobility Management Strategies for the Next Decades. Proceedings of the 82nd Transportation Research Board Annual Meeting, TRB,

Washington, D.C. Disponível em <

http://www.ltrc.lsu.edu/TRB_82/TRB2003-001703.pdf >. Acesso em: 14 jul de 2014. 